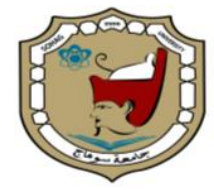

Sohag University

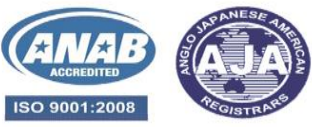

Sohag Medical Journal

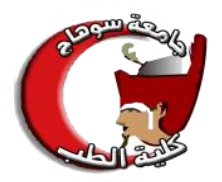

Faculty of Medicine

\title{
Role of Wolbachia in treatment and control of lymphatic filariasis and onchocerciasis
}

\begin{abstract}
Asmaa kamal Abd Ellah.
Medical Parasitology Department, Faculty of Medicine, Sohag University.

Abstract

Both Lymphatic filariasis and onchocerciasis represent serious health issues in tropical regions. Filarial nematodes are the causative agents and these filarial nematodes harbor intracellular alpha-proteobacteria, Wolbachia, that was first observed almost 30 years ago. Wolbachia has evolved a mutualistic symbiosis with these nematodes which are required for their embryogenesis. The essential role of these bacteria in the biology of the nematode makes Wolbachia a promising novel chemotherapeutic target for the treatment of filarial infection. anti-Wolbachia targeting appears a promising alternative for filariasis treatment in situations where current programmatic strategies fail or are unable to be delivered. Wolbachia has been found worldwide in numerous arthropods in addition to filarial nematodes. Wolbachia is a biological method for manipulating the mosquito population and reducing the transmission of disease and increasing the burden on human health. In mosquitoes, the sterility of males can be achieved through, Wolbachia cytoplasmic incompatibility. Thus, the use of Wolbachia bacteria offers a potential strategy for the control of filarial infection.
\end{abstract}

KEYWORDS: Wolbachia, lymphatic filariasis, onchocerciasis, treatment, control.

\section{INTRODUCTION}

The filarial nematodes that cause lymphatic filariasis (LF) or elephantiasis and onchocerciasis or African river blindness affect more than 200 million people throughout the tropics. These nematodes are transmitted by vectors. Because of the high morbidity of these parasitic infections, mass drug administration (MDA) programs were found to overcome them (1). Human LF is caused by Wuchereria bancrofti, Brugia malayi and $\mathrm{B}$. timori and is transmitted by Anopheles and Culex, while human onchocerciasis is caused by Onchocerca volvulus and is transmitted by black flies (Simulium species) (2).

Because of the drawbacks of current therapy for onchocerciasis and LF, a new treatment is needed. Chemotherapy agents targeting Wolbachia, bacteria present in filarial nematodes have been extensively studied (3).

In addition to Wolbachia open a new window to design a strategy to control mosquitoes and vector-borne diseases (4).

\section{Wolbachia: A bacterial endosym- biont}

In 1924, Hertig and Wolbach discovered the endosymbiont bacteria in Culex and these bacteria were named Wolbachia (5). It is maternally inherited alpha proteobacteria, a member of the Rickettsiales order, and presents in arthropod in addition to filarial nematodes such as Wuchereria bancrofti, Brugia malayi, and Onchocerca volvulus (6). 
In the filarial nematode, these bacteria are found in all life stages but vary in numbers between them (7).

Usually, they are found in the hypodermmal cells of the lateral cords of filariae that appear into the body cavity in both males and females (8).

They are also found in the ovaries, oocytes, and embryonic development in females. But they are never found in the male genital system (9).

Furthermore, they are transmitted in these parasitic worms via oocytes to their offsprings (10).

There is a mutualistic relationship between these bacteria and their nematode hosts. As they are essential for the growth, fertileity, and viability of their hosts, while their hosts provide them with amino acids necessary for development (11).

The Absence of these bacteria will affect the viability of the filarial worms (12). All these reasons make them a target for filarial drug treatment and eliminating these parasites (13).

In arthropods, these bacteria make reproductive changes by cytoplasmic incomepatibility in order to maintain themselves and $70 \%$ of insect species seem to carry these bacteria (14).

These bacteria are consisted of at least seven supergroups and a number of additional lineages depend on $16 \mathrm{~S}$ rRNA surface protein (15).

Two subgroups (C and D) present in the nematode hosts while four subgroups (A, $\mathrm{B}, \mathrm{E}, \mathrm{H})$ are found in the arthropods (16). An additional Supergroup (F) appears in both arthropod and nematode and is considered a recent horizontal transfer between the two hosts (17).

\section{Anti-Wolbachia therapy}

Current mass drug administration programs are found to treat annually the most endemic populations with lymphatic fila- riasis and onchocerciasis by using diethylcarbamazine, ivermectin, and albendazole. (18).

These drugs are potent microfilaricides and decrease microfilaria loads in blood and skin but have restricted macrofilaricidal effects. As adults are long-lived and fertile for most of their lives, so patients must be treated for a long time (19).

Furthermore, drug resistance of filarial worms has been demonstrated in several studies (20).

In addition to the long duration of treatment; 17 years for the treatment of onchocerciasis and five years for the treatment of LF and any discontinuation of the treatment will impede the prevention of parasitic transmission. But the treatment of these bacteria has a potent macrofilaricidal effect and also prevents embryoenesis causing the microfilaricidal effect. These properties make these treatments better than current treatments that work against the microfilariae but have limited activity against adults (13).

The destruction of these bacteria causes the death of adult germline cells and the somatic cells in the embryos and microfilariae and results in sterility of these worms (12).

Doxycycline has been established as an effective drug against these bacteria with good macrofilaricidal activity and has been recommended as a therapy for lymphatic filariasis and onchocerciasis (21).

Although this drug is readily available, cheap, safe to use and effective macrofilaricidal drugs, several limitations impeded its use in MDA programs (22).

It needs a long course of treatment (4-6 weeks) and is contraindicated for children and pregnant or breast-feeding women (23).

Therefore, the anti-Wolbachia consortiu$\mathrm{m}$ (A-WOL) was established in 2007 and aimed to recognize new suitable drugs ag- 
ainst these bacteria that present in filarial worms which are used in the contraindicated groups, with short courses and could be used on a wide-scale (24).

In order to improve doxycycline treatment; the addition of a second antibiotic; rifampicin was done. This combination therapy decreased the length of treatment in murine filariasis (25).

Another factor that improved the mode of delivery of doxycycline to increase the absorption of the drug, was the use of liposomes form of tetracycline to treat murine filariasis. This form of the drug was applied subcutaneously to infected rodents and the effects of microfilaricidal and macrofilaricidal effects were determined and compared with the therapy of free tetracycline that was applied orally to rodents. The liposome form was more efficient than the free tetracycline (26).

\section{Wolbachia as Eco-Friendly Biocontrol Agent}

At present, several strategies that do not cause any harm to the environment are achieved to diminish the transmission of the disease, such as the strategy of releasing sterile males in the environment male mosquitoes were sterilized by exploiting the phenomenon of cytoplasmic incompatibility instead of irradiation (27).

The sterility of male mosquitoes can be achieved through the infection of mosqueitoes with Wolbachia (28).

These bacteria cause embryonic death in mosquitoes called cytoplasmic incompatibility (29).

This phenomenon is due to the incomepatibility of the sperm and eggs in mosqueitoes that make these bacteria strongly invade these insects (30).

Release mosquitoes infected with these bacteria in natural populations is an important measure to control diseases transmitted by mosquitoes (31)
In addition to Wolbachia decrease the transmission of diseases through insects by two methods, the first method is by inhibiting the proliferation of pathogens in vectors and the second method is by reducing the life expectancy of vectors (32).

\section{Conclusions}

The filarial diseases cause global disability despite MDA programs present. Wolbachia is a promising therapy against filariasis, and antibiotics that target these bacteria are effective than standard treatments due to their long-lasting effects and macrofilaricidal activity. Also, these bacteria are a biological method to control mosquitoes and reducing the transmitssion of disease. We recommend that future research work should be directed toward Wolbachia treatment and Wolbachia-based control strategies.

\section{REFERENCES}

1. Molyneux DH, Bradley M, Hoerauf A, Kyelem D, and Taylor MJ. Mass drug treatment for lymphatic filariasis and onchocerciasis. Trends Parasitol; 2003; 19: 516-522.

2. Muller R and Wakelin D. Worms and human disease. CABi; 2002; 188-211.

3. Hoerauf, A. New strategies to combat filariasis. Expert review of anti-infect Ther; 2006; 4: 211-222.

4. Chegeni TN and Fakhar M. Promising Role of Wolbachia as Anti-parasitic Drug Target and Eco-Friendly Biocontrol Agent. Recent patents on anti-inf. drug discovery; 2019; 14: 69-79.

5. Hertig, M. and Wolbach, S.B. Studies on rickettsia-like micro-organisms in insects. J Med Res;1924; 44, 329.

6. Williams KP, Sobral BW and Dickerman AW. A robust species tree for the alphaproteobacteria. J Bacteriol; 2007; 189: 4578-4586.

7. McGarry HF, Egerton GL and Taylor MJ. Population dynamics of Wolbachia 
bacterial endosymbionts in Brugia malayi. Mol Biochem Parasitol; 2004; 135: 57-67.

8. Hoerauf A, Mand S, Volkmann L, Büttner M, Marfo-Debrekyei Y, Taylor $M$, Adjei $O$ and Büttner DW. Doxycycline in the treatment of human onchocerciasis: kinetics of Wolbachia endobacteria reduction and of inhibition of embryogenesis in female Onchocerca worms. Microbes Infect; 2003; 5: 261273.

9. Fischer K, Beatty WL, Jiang D, Weil GJ and Fischer,PU. Tissue and stagespecific distribution of Wolbachia in Brugia malayi. PLoS Negl Trop Dis; 2011; 5:1174.

10.Taylor MJ, Bilo K, Cross HF, Archer JP and Underwood AP. 16S rDNA Phylogeny and Ultrastructural Characterization of Wolbachia Intracellular Bacteria of the Filarial Nematodes Brugia malayi, B. pahangi, and Wuchereria bancrofti. Exp parasitol; 1999; 91: 356-361.

11.Foster J, Ganatra M, Kamal I, Ware J, Makarova K, Ivanova N, Bhattacharyya A, Kapatral V, Kumar $\mathbf{S}$ and Posfai J. The Wolbachia genome of Brugia malayi: endosymbiont evolution within a human pathogenic nematode. PLos biol; 2005; 3: e121.

12.Landmann F, Voronin D, Sullivan W and Taylor MJ. Anti-filarial activity of antibiotic therapy is due to extensive apoptosis after Wolbachia depletion from filarial nematodes. PLoS Pathog; 2011; 7: e1002351.

13. Taylor MJ, Hoerauf $A$ and Bockarie $M$. Lymphatic filariasis and onchocerciasis. Lancet; 2010; 376: 1175-1185.

14. Werren JH, Baldo $L$ and Clark ME. Wolbachia: master manipulators of invertebrate biology. Nat Rev Microbiol; 2008; 6: 741-751.

15.Bordenstein SR, Paraskevopoulos C, Dunning Hotopp JC, Sapountzis P, Lo $\mathrm{N}$, Bandi C, Tettelin H, Werren JH and Bourtzis K. Parasitism and mutualism in Wolbachia: what the phylogenomic trees can and cannot say. Mol Biol evol; 2009; 26: 231-241.

16.Bandi C, Anderson, TJ, Genchi C. and Blaxter ML. Phylogeny of Wolbachia in filarial nematodes. Proc Royal Soc London Series B Biol Sci; 1998; 265:2407-2413.

17. Casiraghi M, Bordenstein S, Baldo $\mathbf{L}$, Lo $\mathbf{N}$, Beninati $\mathbf{T}$, Wernegreen $J$, Werren J, and Bandi C. Phylogeny of Wolbachia pipientis based on gltA, groEL and ftsZ gene sequences: clustering of arthropod and nematode symbionts in the F supergroup, and evidence for further diversity in the Wolbachia tree. Microbio; 2005; 151: 4015-4022.

18. Hoerauf A, Pfarr K, Mand S, Debrah A and Specht S. Filariasis in Africa treatment challenges and prospects. Clin Microbio Infect; 2011; 17: 977-985.

19.Stolk WA, De Vlas SJ, Borsboom GJ and Habbema JD. LYMFASIM, a simulation model for predicting the impact of lymphatic filariasis control: quantification for African villages. Parasitol; 2008; 135: 1583-1598.

20.Osei-Atweneboana MY, Awadzi K, Attah SK, Boakye DA, Gyapong JO and Prichard RK. Phenotypic evidence of emerging ivermectin resistance in Onchocerca volvulus. PLoS Negl Trop Dis; 2011; 5:998.

21.Mand S, Debrah AY, Klarmann U, Batsa L, Marfo-Debrekyei Y, Kwarteng A, Specht S, Belda-Domene $A$, Fimmers $\mathbf{R}$ and Taylor $M$. Doxycycline improves filarial lymphedema independent of active filarial infection: a randomized controlled trial. Clin Infect Dis; 2012; 55: 621-630.

22. Walker M, Specht S, Churcher TS, Hoerauf A, Taylor MJ and Basáñez MG. Therapeutic efficacy and macrofilaricidal activity of doxycycline for the treatment of river blindness. Clin Infect Dis; 2015; 60: 1199-1207.

23.Hansen RD, Trees AJ, Bah GS, Hetzel $\mathrm{U}$, Martin C, Bain O, Tanya VN and Makepeace BLA worm's best friend: recruitment of neutrophils by Wolbachia 
confounds eosinophil degranulation against the filarial nematode Onchocerca ochengi. Proc Royal Soc Biol Sci; 2011; 278: 2293-2302.

24.A-WOL. Anti Wolbachia Consortium.2016; https://awol.lstmed.ac.uk.

25. Volkmann L, Fischer K, Taylor M and Hoerauf AAntibiotic therapy in murine filariasis (Litomosoides sigmodontis): comparative effects of doxycycline and rifampicin on Wolbachia and filarial viability. Trop Med Int Health; 2003; 8: 392-401.

26.Bajpai P, Vedi S, Owais M, Sharma, SK, Saxena PN and MisraBhattacharya S. Use of liposomized tetracycline in elimination of Wolbachia endobacterium of human lymphatic filariid Brugia malayi in a rodent model. $\mathbf{J}$ Drug Target; 2005;13: 375-381.

27.Lees RS, Gilles JR, Hendrichs J, Vreysen MJ and Bourtzis K. Back to the future: the sterile insect technique against mosquito disease vectors. Curr Opin Insect Sci; 2015; 10, 156-162.33
28.Supali T, Djuardi Y, Pfarr KM, Wibowo H, Taylor MJ, Hoerauf A, Houwing-Duistermaat JJ, Yazdanbakhsh $M$ and Sartono $E$. Doxycycline treatment of Brugia malayi infected persons reduces microfilaremia and adverse reactions after diethylcarbamazine and albendazole treatment. Clin Infect Dis; 2008;46: 13851393.

29.Hoffmann AA. Incompatible mosquitoes. Nature; 2005; 436: 189-189.

30.Kriesner P, Hoffmann AA, Lee SF, Turelli $M$ and Weeks AR. Rapid sequential spread of two Wolbachia variants in Drosophila simulans. PLoS. Pathog; 2013; 9: 1003607.

31.Benedict MQ and RobinsonbAS. The first releases of transgenic mosquitoes: an argument for the sterile insect technique. Trends Parasitol; 2003; 19: 349-355.

32.McMeniman CJ and $O^{\prime}$ Neill SL. A virulent Wolbachia infection decreases the viability of the dengue vector Aedes aegypti during periods of embryonic quiescence. PLoS Negl Trop Dis; 2010; 4 : e748. 